\title{
Erratum to: TMEM70 deficiency: long-term outcome of 48 patients
}

\author{
Martin Magner • Veronika Dvorakova • Marketa Tesarova • Stella Mazurova • Hana Hansikova • \\ Martin Zahorec • Katarina Brennerova • Vladimir Bzduch • Ronen Spiegel • Yoseph Horovitz • \\ Hanna Mandel • Fatma Tuba Eminoğlu • Johannes Adalbert Mayr • Johannes Koch • \\ Diego Martinelli • Enrico Bertini • Vassiliki Konstantopoulou • Joél Smet • Shamima Rahman • \\ Alexander Broomfield • Vesna Stojanović • Carlo Dionisi-Vici • Rudy van Coster • \\ Eva Morava • Wolfgang Sperl • Jiri Zeman • Tomas Honzik
}

Published online: 17 March 2015

(C) SSIEM 2015

\section{Erratum to: J Inherit Metab Dis}

DOI 10.1007/s10545-014-9774-8

The correct name of the contributing author Eva Morava-

Kozicz is Eva Morava.

The online version of the original article can be found at http://dx.doi.org/ 10.1007/s10545-014-9774-8.

M. Magner $\cdot$ V. Dvorakova $\cdot$ M. Tesarova $\cdot$ S. Mazurova $\cdot$

H. Hansikova $\cdot$ J. Zeman $\cdot$ T. Honzik $(\bowtie)$

Department of Pediatrics and Adolescent Medicine, First Faculty of Medicine, Charles University in Prague and General University

Hospital in Prague, Ke Karlovu 2, 12808 Prague 2, Czech Republic

e-mail: Tomas.Honzik@vfn.cz

\section{Zahorec}

Cardiac Intensive Care Unit, Pediatric Cardiac Center, National Institute of Cardiovascular Diseases, Limbova 1, 833

51 Bratislava, Slovakia

K. Brennerova $\cdot$ V. Bzduch

I. Department of Pediatrics Comenius, University Children's

Hospital, Bratislava, Limbova 1, 83340 Bratislava, Slovakia

\section{R. Spiegel $•$ Y. Horovitz}

Department of Pediatrics, Emek Medical Center, Rappaport Faculty of Medicine, Technion, Haifa, Rabin St., Afula 1810100, Israel

H. Mandel

Department of Pediatrics, Metabolic Unit, Rambam Medical Center, Rappaport Faculty of Medicine, Technion, Haifa 31906, POB 9602, Israel
F. T. Eminoğlu

Department of Pediatric Metabolism, Ankara University School of Medicine, 06100 Cebeci, Ankara, Turkey

J. A. Mayr · J. Koch $\cdot$ W. Sperl

Department of Pediatrics, Paracelsus Medical University Salzburg, Müllner Hauptstraße 48, A-5020 Salzburg, Austria

D. Martinelli • C. Dionisi-Vici

Unit of Metabolism, Bambino Gesù Children's Hospital, IRCCS,

Piazza Sant'Onofrio, 4 - 00165 Rome, Italy

E. Bertini

Unit for Neuromuscular and Neurodegenerative Disorders, Laboratory of Molecular Medicine, Bambino Gesù Children's Hospital, IRCCS, Piazza Sant'Onofrio, 4 - 00165 Rome, Italy

V. Konstantopoulou

Department of Pediatrics and Adolescent, Medical University of Vienna, Medicine, Währinger Gürtel 18-20, A-1090 Vienna, Austria 
J. Smet · R. van Coster

Department Pediatrics, Division of Pediatric Neurology and Metabolism, Mitochondrial University Hospital Ghent, Investigations Laboratory, 3K5, De Pintelaan,

185-9000 Ghent, Belgium

S. Rahman $\cdot$ A. Broomfield

Metabolic Department, Great Ormond Street Hospital, London WC1N 3JH, UK

S. Rahman • A. Broomfield

Genetics and Genomic Medicine, UCL Institute of Child Health, London WC1N 1EH, UK

\section{Stojanović}

Institute for Child and Youth Health Care of Vojvodina, Intensive Unit Care, Medical Faculty, University of Novi Sad, Hajduk Veljkova 10, 21000 Novi Sad, Serbia

E. Morava

Nijmegen Center for Mitochondrial Disorders (NCMD) at the Department of Pediatrics and the Institute of Genetic and Metabolic Disease (IGMD), Radboud University Medical Centre, P.O Box 9101, 6500 HB Nijmegen, The Netherlands 